\title{
Icariside II ameliorates diabetic nephropathy in streptozotocin-induced diabetic rats
}

This article was published in the following Dove Press journal:

Drug Design, Development and Therapy

7 September 2015

Number of times this article has been viewed

\section{Wenjie Tian ${ }^{1,2, *}$ \\ Hongen Lei ${ }^{1, *}$ \\ Ruili Guan' \\ Yongde $\mathrm{Xu}^{\prime}$ \\ Huixi Li' \\ Lin Wang' \\ Bicheng Yang' \\ Zhezhu Gao' \\ Zhongcheng Xin'}

'Andrology Center, Peking University First Hospital, Peking University, Beijing, ${ }^{2}$ Department of Urology, The Second Hospital of Jilin University, Jilin University, Changchun, People's Republic of China

*These authors contributed equally to this work
Correspondence: Zhongcheng Xin Andrology Center, Peking University First Hospital, Peking University, No 8, Xishiku St, Xicheng District, Beijing 100034, People's Republic of China

Tel +861083228989

Fax +86 1083222822

Email xinzc@bjmu.edu.cn
Purpose: To investigate the therapeutic effects and potential mechanisms of icariside II (ICA II) on reversing diabetic nephropathy in streptozotocin (STZ)-induced type I diabetic rats.

Methods: Newborn male Sprague Dawley rats were labeled with thymidine analog 5-ethynyl-2deoxyuridine (EdU) for tracking endogenous label retaining progenitor cells (LRCs). At age of 8 weeks, 48 rats were randomly divided into three groups: normal control group $(n=16)$, diabetes mellitus group (DM; $n=16$ ), and diabetes mellitus plus ICA II therapy group (DM+ICA II, n=16). Eight weeks induced for diabetes with STZ, rats in DM group and DM+ICA II group were treated with vehicle or ICA II ( $5 \mathrm{mg} / \mathrm{kg} /$ day) for another 8 weeks, respectively. Then, blood creatinine, 24-hour urine protein, blood urea nitrogen, and glycosylated hemoglobin were measured, as well as the expression of von Willebrand factor, malondialdehyde, transforming growth factor- $\beta$ / drosophila mothers against decapentaplegic protein/connective tissue growth factor (TGF- $\beta$ / Smad/CTGF) signaling, marker of proliferation Ki-67, and EdU+ LRCs in renal tissues.

Results: Increased levels of creatinine, 24-hour urine protein, and blood urea nitrogen and remarkably decreased proportion of normal glomeruli and increased proportions of I, IIa, IIb, and III glomeruli were observed in diabetic rats, while ICA II could reverse these changes. Interestingly, ICA II could significantly downregulate the levels of malondialdehyde and TGF- $\beta / \mathrm{Smad} / \mathrm{CTGF}$ signaling and increase the expression of von Willebrand factor, Ki-67, and EdU+ LRCs in the kidney.

Conclusion: ICA II treatment could ameliorate diabetic nephropathy in STZ-induced diabetic rats by increasing endothelial cell contents, downregulating TGF- $\beta / \mathrm{Smad} / \mathrm{CTGF}$ signaling pathway and oxidative stress level, and promoting cell proliferation both in kidney cortex and medulla. These beneficial effects appear to be mediated by its antioxidant capacity and recruitment of endogenous EdU+ progenitor cells into the kidney tissue.

Keywords: diabetic nephropathy, icariside II, EdU, diabetes mellitus, label retaining progenitor cells

\section{Introduction}

Diabetic nephropathy (DN), a major complication of diabetes mellitus (DM) in both type I and type II, is a leading cause of end-stage renal disease in the clinic. Progression of DN is featured as glomerular hypertrophy, expansion of the mesangial matrix, and thickening of glomerular and tubular basement membranes. ${ }^{1,2}$ The hallmark of proteinuria, glomerulosclerosis, and tubulointerstitial fibrosis is highlighted in later stages of the disease. Although a number of key factors such as oxidative stress, ${ }^{3}$ glucose, ${ }^{4}$ advanced glycation end products, ${ }^{5}$ glomerular hypertension, ${ }^{6}$ and various signal transduction have been implicated in the pathogenesis of $\mathrm{DN}$, however, the exact mechanisms involved in this complex process have not been well elucidated; therefore, no specific therapies for this condition have been developed. 
It is putative that various signaling pathways mutually take part in the progression of DN. Transforming growth factor- $\beta$ (TGF- $\beta$ ) signaling pathway, as one of the key factors, has been shown to play a critical role, which regulates the extracellular matrix (ECM) accumulation of kidney to promote the renal glomerulosclerosis and inflammation in diabetic rat model. ${ }^{7}$ It is well known that drosophila mothers against decapentaplegic protein (Smad) is one of the downstream signaling molecule of TGF- $\beta$, and phosphorylation of Smad 2 and Smad3 is associated with the expression of fibronectin and collagen IV in the DN. ${ }^{8}$ Interestingly, connective tissue growth factor (CTGF) also acts the downstream molecule of TGF- $\beta$ and regulates the invasive new vessel formation and plays a role in angiogenesis and fibrosis in the DN.,10

Many recent studies have demonstrated that stem cell therapy was a powerful tool to prevent and treat DN in diabetic rat models. ${ }^{11}$ Mesenchymal stem cells (MSCs) treatment ameliorates podocyte injury and proteinuria and has been shown to be effective in DN. ${ }^{12,13}$ However, insufficient source, invasive procedures, ethical, and regulatory issues hampered their use in clinical applications. Fortunately, endogenous stem/progenitor cells resident in organs and tissues play critical roles for organogenesis during development and for tissue homeostasis in adulthood. It is worth to note that even without any therapeutic intervention, human body possesses a robust self-healing capability to repair damaged tissues or organs. Therefore, stem cells for DN therapy should not be limited to a supply side approach; the resident endogenous stem cells existing in patients could also be a potential target for DN therapy. Actually, many studies indicated that the recruitment of MSCs plays an pivotal role in the progression of DN. ${ }^{14}$ Very recently, to localize and track the endogenous progenitor cells, label retaining progenitor cell (LRC) strategy has been successful used in investigating the effects of low-energy shockwave therapy on improving erectile function and tissue damages in a streptozotocininduced diabetic (STZ-DM) rats model. ${ }^{15}$

Herba Epimedii, a traditional Chinese medicine, has been extensively utilized for the treatment of many diseases including sexual dysfunction, ${ }^{16,17}$ osteoporosis, ${ }^{18}$ cardiovascular diseases, ${ }^{19}$ and cancer. ${ }^{20,21}$ Icariin is one of the most abundant flavonoids in Herba Epimedii ${ }^{22}$ and has been demonstrated a protective effect on the early stage of experimental DN in STZ-DM rats via modulating TGF- $\beta$ in rats, suggesting the important role of TGF- $\beta /$ Smad signaling in the benefit of DN. ${ }^{23}$ Icariside II (ICA II, $\mathrm{C}_{27} \mathrm{H}_{32} \mathrm{O}_{10}$, molecular weight [MW] $514.54 \mathrm{kDa}$ ), the bioactive form of icariin, lacks a glucose moiety at C-7 and is more bioavailable than icariin. ${ }^{24}$ In this study, thymidine analog 5-ethynyl-2-deoxyuridine (EdU) was used for tracking the putative endogenous stem cells in the kidney. Furthermore, we investigated the feasibility and underlying mechanisms of ICA II in reversing DN in a rat model of type I DM.

\section{Materials and methods}

\section{Animals and treatments}

All animal experiments in this study were approved by the Institutional Animal Care and Use Committee at our institution. Newborn Male Sprague Dawley rats were intraperitoneally injected with EdU (50 mg/kg, Invitrogen, Carlsbad, CA, USA) immediately within the first day after birth. At age of 8 weeks, 48 rats were randomly divided into three groups: normal control (NC, $n=16$ ) group, diabetic group (DM, $n=16$ ), and diabetic plus ICA II therapy group (DM+ICA II, $n=16$ ). The NC rats and the diabetic rats were injected intraperitoneally with blank solvent $(0.1 \mathrm{~mol} / \mathrm{L}$ citrate-phosphate buffer, $\mathrm{pH} 4.5$ ) and $60 \mathrm{mg} / \mathrm{kg}$ of STZ (Sigma-Aldrich, St Louis, MO, USA), respectively. The success of diabetic model was confirmed by blood glucose levels ( $\geq 16.7 \mathrm{mmol} / \mathrm{L}$ ) 72 hours later and throughout the study. After STZ injection, only 16/16, $12 / 16$, and $13 / 16$ rats met the glucose criteria in NC, DM, and DM+ICA II groups, respectively, followed by standard husbandry care feeding continued for 8 weeks. Then, vehicle treatment (dimethyl sulfoxide) for NC group and DM group rats or ICA II treatment ( $5 \mathrm{mg} / \mathrm{kg} /$ day) for DM+ICA II group rats by gavage feeding were administered for another 8 weeks. Finally, 15/16, 10/12, and 11/13 rats survived to the end of study and 12/15,8/10, and 9/11 enough blood samples were successfully obtained due to the hard procedure in NC, DM, and DM+ICA II group, respectively. Hence, eight random samples of rats from NC, DM, and DM+ICA II groups were performed at the end of the study.

\section{Measurements for biochemical indexes}

Eight random blood samples of rats from NC, DM, and DM+ICA II groups were performed to detect glycosylated hemoglobin $\left(\mathrm{HbA}_{1 \mathrm{c}}\right)$, 24-hour urine protein, creatinine $(\mathrm{Cr})$, and blood urea nitrogen (BUN) by the clinical laboratory in our hospital according to the manufacturer's instructions. The activity of malondialdehyde (MDA) concentration was measured with the thiobarbituric acid (TBA) reaction method. Briefly, the supernatant fraction of kidney tissue homogenate was mixed with TBA reagent consisting of $0.375 \%$ TBA and $15 \%$ trichloroacetic acid in $0.25 \mathrm{mM}$ hydrochloric acid. The reaction mixtures were placed in boiling water, and then the absorbance of the supernatant was measured at $535 \mathrm{~nm}$. The 
bicinchoninic acid assay was used for protein quantization. MDA levels were expressed as $\mu \mathrm{mol} / \mathrm{mg}$ protein.

\section{Immunohistochemistry and periodic acid-Schiff staining}

Freshly dissected kidney tissue was fixed for 4 hours with cold $2 \%$ formaldehyde and $0.002 \%$ picric acid in $0.1 \mathrm{M}$ phosphate buffer and were subsequently embedded in paraffin and cut into $4-\mu \mathrm{m}$ sections using a rotor microtome. The slices were then washed in phosphate-buffered saline (PBS) for 5 minutes and were incubated in $0.1 \%$ Triton X-100 and 5\% goat serum for 30 minutes. Then, the sections were incubated with anti-endothelial cell antibody (1:200, Abcam, Cambridge, MA, USA) overnight at $4^{\circ} \mathrm{C}$ in a humidified chamber. The slides were subsequently incubated with the secondary antibody using the MaxVision TM horseradish peroxidase-polymer anti-rabbit immunohistochemistry (IHC) kit (Maixin Company, Fuzhou, People's Republic of China). Next, we used the paraffin sections for periodic acid-Schiff (PAS) staining (Loogene Biotechnology Company Ltd., Beijing, People's Republic of China). Glomerulosclerosis was quantified on PAS-stained sections based on four hierarchical glomerular lesions with a separate evaluation for degrees of interstitial and vascular involvement as described previously (The descriptions of four classes of glomerular lesions in DN are class I, mild or nonspecific light microscopy changes and electron microscopy-proven glomerular basement membrane thickening; class IIa, mild mesangial expansion; class IIb, severe mesangial expansion; class III, nodular sclerosis [Kimmelstiel-Wilson lesion]; and class IV, advanced diabetic glomerulosclerosis). ${ }^{25}$ The assessments were independently carried out by two researchers in a blinded manner.

\section{Immunofluorescence and EdU staining}

Freshly harvested kidney tissues were fixed for 4 hours with cold $2 \%$ formaldehyde and $0.002 \%$ picric acid in $0.1 \mathrm{M}$ phosphate buffer, followed by overnight immersion in buffer solution containing $30 \%$ sucrose. Tissues were then frozen in optimum cutting temperature compound (Sakura Finetek, Torrance, CA, USA) and stored at $-80^{\circ} \mathrm{C}$ until use. Sections were cut at $5 \mu \mathrm{m}$, adhered to charged slides, and subjected to immunofluorescence and EdU staining.

For immunofluorescence staining, the slices were then washed in PBS for 5 minutes and were incubated in $0.1 \%$ Triton X-100 and 5\% goat serum for 30 minutes. Primary antibodies was rabbit anti-von Willebrand factor (vWF) antibody (1:200, Abcam) and rabbit anti-marker of proliferation Ki-67 (Ki67) antibody (1:200, Abcam). After rinsing thrice with PBS, the sections were incubated with Texas Red-conjugated secondary antibody (Vector Laboratories, Burlingame, CA, USA). Nuclear staining was performed with 4',6-diamidino-2-phenylindole (DAPI).

EdU staining was performed according to the instruction of Click-IT reaction cocktail (Invitrogen, Carlsbad, CA, USA). In brief, frozen tissue sections $(5 \mu \mathrm{m})$ were placed $0.3 \%$ Triton X-100 in PBS for 20 minutes. After rinsing twice with PBS, the tissue sections were incubated with the cocktail reagents for 30 minutes at room temperature. Then nuclear staining was carried out with DAPI after washing twice with PBS.

\section{Western blotting}

The renal tissue was homogenized in lysis buffer (Keygen Biotech, Nanjing, People's Republic of China) on ice for 15 minutes. Protein samples at $20 \mu \mathrm{g}$ were loaded onto the $10 \%$ SDS-PAGE gel and then transferred onto polyvinylidenefluoride (PVDF) membranes. Anti-TGF- $\beta 1$ (1:1,000, Abcam), anti-Smad2 (1:1,000, Abcam), anti-phospho-Ser467-Smad2 (1:500, Abcam), anti-CTGF (1:500, Abcam), and anti-GAPDH (1:1,000, Abcam) antibodies were used to incubated with the membranes overnight at $4^{\circ} \mathrm{C}$. Then horseradish peroxidase-conjugated secondary antibodies incubated the membranes for 1 hour at room temperature. Enhanced chemiluminescence regents (Immobilon Western, EMD Millipore, Billerica, MA, USA) were used, and data were collected by C-digit machine (LI-COR Biosciences, Cambridge, UK).

\section{Image analysis and quantification}

Images of immunofluorescence and EdU staining were captured using a digital camera (DMI6000B, Leica Microsystems, Nussloch, Germany), and images of IHC and PAS staining were captured using a digital camera (DM2500, Leica Microsystems); then images were imported into ImagePro plus software (version 6.0, Media Cybernetics Inc., Bethesda, MD, USA) for calculating the mean density and dot numbers of each sample. The integrated density value of each protein band of Western blotting images was analyzed with ImageJ software (version 1.46r, ImageJ, National Institutes of Health, Bethesda, MD, USA).

\section{Statistical analysis}

Statistical analysis was performed with the SPSS 17.0 software. Data were expressed as mean \pm standard deviation. Multiple groups were compared using one-way analysis of variance followed by the Tukey honest significance test post 
hoc comparisons. A two-group comparison was done using $t$-test, and $P<0.05$ was considered significant.

\section{Results}

\section{ICA II improved kidney functional indexes in STZ-DM rats}

The body weight, blood glucose level, 24-hour urine protein, $\mathrm{Cr}, \mathrm{BUN}$, and $\mathrm{HbA}_{1 \mathrm{c}}$ of the rats are presented in Table 1. The body weights of the STZ-induced rats were significantly lower than those in the $\mathrm{NC}$ group. In addition, the serum glucose, 24-hour urine protein, $\mathrm{Cr}, \mathrm{BUN}$, and $\mathrm{HbA}_{1 \mathrm{c}}$ in the DM group were significantly higher compared with those in the NC group. However, no significant differences in body weight, blood glucose level, and $\mathrm{HbA}_{1 \mathrm{c}}$ levels were found between the DM group and DM+ICA II group. Conversely, diabetic rats treated with ICA II showed an attenuated renal function injury (24-hour urine protein, $\mathrm{Cr}$, and BUN) in comparison with the STZ-induced DM group rats (Table 1).

\section{Glomerular lesions in renal tissues were improved by ICA II}

The kidney diagnosed as DN are classified for four progressive types of class I, IIa, IIb, III, and IV grade based on glomerular lesions, with a separate evaluation for interstitial and vascular lesions as described by Tervaert et al. ${ }^{25}$ There are no class IV grades of DN in our present model. Quantitative analyses showed that the percentage of normal glomerulus in the diabetic rats remarkably decreased, and the proportion of I, IIa, IIb, and III glomerulus significantly increased compared with the NC group. Moreover, administration of ICA II in diabetic rats remarkably improved the percentage of normal glomerulus and decreased the proportion of class I, IIa, IIb, and III grade compared with the DM group (Figure 1).

Table I Effects of ICA II on serum, urine biochemical parameters, and body weights in NC, DM, and DM+ICA II rats

\begin{tabular}{|c|c|c|c|}
\hline Variables & $N C(n=8)$ & $D M(n=8)$ & $\begin{array}{l}\text { DM+ICA II } \\
(n=8)\end{array}$ \\
\hline Body weights (g) & $501.38 \pm 23.01$ & $225.8 \pm 18.2 *$ & $234.49 \pm 24.49 *$ \\
\hline $\begin{array}{l}\text { Serum glucose } \\
(\mathrm{mmol} / \mathrm{L})\end{array}$ & $8.2 \pm 0.62$ & $29.3 \pm 3.27^{*}$ & $32.49 \pm 3.25 *$ \\
\hline $\begin{array}{l}\text { 24-hour urine protein } \\
(\mathrm{mg} / 24 \mathrm{~h})\end{array}$ & $13.84 \pm 3.0$ & $73.16 \pm 5.56 *$ & $41.13 \pm 5.63^{* \#}$ \\
\hline $\mathrm{Cr}(\mu \mathrm{mol} / \mathrm{L})$ & $52.4 \pm 9.37$ & $88.8 \pm 10.47^{*}$ & $64.8 \pm 7.66^{*, \#}$ \\
\hline BUN (mmol/L) & $7.22 \pm 0.53$ & $14.29 \pm 1.19 *$ & $8.90 \pm 0.77^{\#}$ \\
\hline $\mathrm{HbA}_{\mathrm{lc}}(\%)$ & $8.7 \pm 2.27$ & $|8.6| \pm 0.4^{*}$ & $16.5 \mid \pm 2.97 *$ \\
\hline
\end{tabular}

Notes: $* P<0.05$ versus $N C$ group, ${ }^{\# P<0.05}$ versus DM group.

Abbreviations: NC, normal control; DM, diabetes mellitus; ICA II, icariside II; $\mathrm{Cr}$, creatinine; $\mathrm{BUN}$, blood urea nitrogen; $\mathrm{HbA}_{\mathrm{lc}}$, glycosylated hemoglobin.

\section{ICA II increased renal endothelial contents in STZ-DM rats}

The expression of vWF, one of the most important endothelial markers, was detected by immunofluorescence staining in renal tissues. Results shows that STZ injection resulted a significant decrease of vWF expression in the DM group compared with the NC group. Significant recovery of the vWF expression was observed in diabetic rats treated with ICA II (Figure 2).

\section{Antioxidant effects of ICA II in diabetes}

STZ induced a significant increase in the level of MDA compared with that in the NC group, suggesting a clear lipid peroxidation in the kidney tissue of STZ-induced diabetic rats. Administration of ICA II significantly attenuated the MDA levels in the kidney tissues of the diabetic rats. However, there was still a significant difference in MDA levels between the $\mathrm{NC}$ group and the DM+ICA II group (Figure 3 ).

\section{Regulating TGF- $\beta /$ Smad/CTGF signaling in STZ-DM rats by ICA II}

Upregulation of TGF- $\beta /$ Smad/CTGF signaling had been implicated in mediating the glomerulosclerosis, matrix expansion, and fibrosis in DN. To evaluate the effects of ICA II on TGF- $\beta /$ Smad/CTGF signaling, Western blotting was performed to evaluate the expression of TGF- $\beta 1, \mathrm{P}-\mathrm{Smad} 2$, Smad2, and CTGF. It was confirmed that TGF- $\beta / \mathrm{Smad} /$ CTGF signaling was activated in the STZ-induced rats compared with that in the $\mathrm{NC}$ group; interestingly, those signaling was significantly suppressed when the diabetic rats were treated with ICA II (Figure 4).

\section{Cell proliferation by the expression of $\mathrm{Ki} 67$}

Cell proliferation in renal tissues was assessed as expression of Ki67. As shown by automated microscopy, proliferation index (Ki67/DAPI) was significantly decreased in the STZinduced DM group when compared with the NC group, whereas ICA II significantly increased the expression of Ki67 in the DM+ICA II group (Figure 5).

\section{EdU labeling and retaining in renal tissues}

LRC is a frequently employed strategy for the identification of resident or migrated stem cells in postnatal tissues. In this study, we used LRC to detect the putative endogenous stem cells by injection of EdU in neonatal animals, followed by tracking EdU in kidney tissues. The data showed that EdU+ cells in the kidney mainly reside in the medulla. The number 

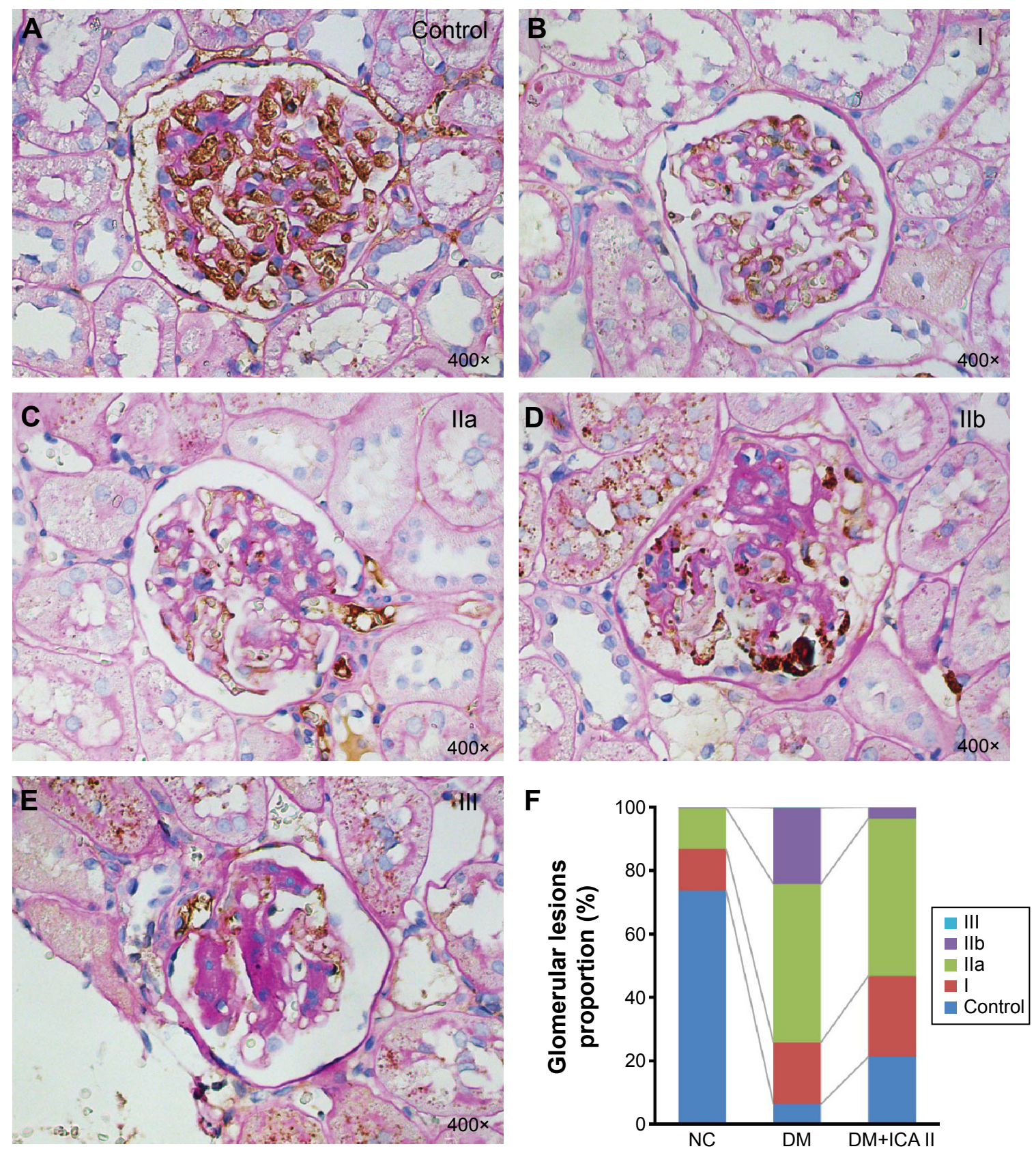

Figure I Effects of icariside II on the glomerular lesion grades.

Notes: Glomerular lesions were examined by IHC for anti-endothelial cell antibody expression and PAS staining. (A-E) Normal glomerulus and four progressive types of class I, Ila, Ilb, and III grade of DN based on glomerular lesions. (F) Quantitative analysis of percentages of glomerular lesions in NC, DM, and DM+ICA II groups is shown in the bar graph ( $n=8$ per group).

Abbreviations: DM, diabetes mellitus; DN, diabetic nephropathy; ICA, icariside; IHC, immunohistochemistry; NC, normal control; PAS, periodic acid-Schiff.

of EdU+ cells in the kidney (medulla and cortex) significantly decreased in the STZ-induced DM group rats when compared with NC, while administration of ICA II could significantly increase the number of EdU+ cells in kidney (Figure 6).

\section{Discussion}

DN is a major diabetic complication that leads to the severe end-stage renal diseases. There is still no effective preventative or therapeutic approaches until now. In this study, a diabetic rat model was established and used for evaluating the therapeutic effects of ICA II on DN and its potential mechanisms. In previous studies, icariin has been demonstrated to attenuate the $\mathrm{Cr}$, urinary albumin excretion, and BUN, but not serum glucose in STZ-DM rats. ${ }^{23}$ It was consistently observed in our present study by ICA II administration, confirming that ICA II alleviates DN by other ways, 

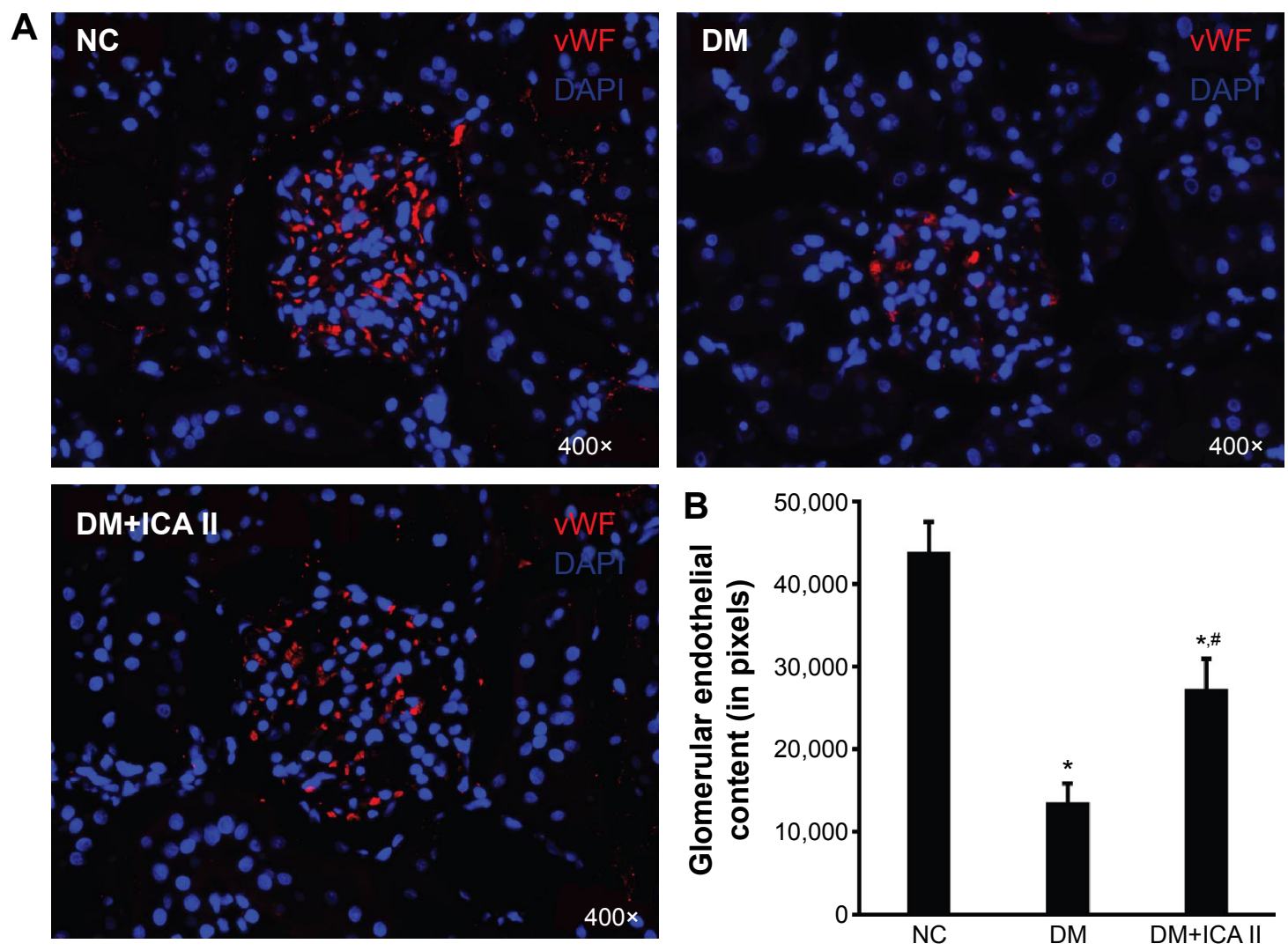

Figure 2 Effects of icariside II on the glomerular endothelial content.

Notes: Kidney tissues were examined by immunofluorescent staining for vWF expression. (A) Compared with that in the NC group, the expression of vWF in the glomerulus of the DM group was decreased. ICA II increased the expression of vWF. (B) Quantative analysis of vWF expression in glomerular endothelial cells is shown in the column bar graph ( $n=8$ per group). $* P<0.05$ versus $N C$ group and ${ }^{*} P<0.05$ versus $D M$ group.

Abbreviations: DAPI, 4',6-diamidino-2-phenylindole; DM, diabetes mellitus; ICA, icariside; NC, normal control; vWF, von Willebrand factor.

but not by lowering glucose. In addition, the concentration of MDA increased in STZ-DM rats model compared with that of the NC group in the current study was consistent with reports from other research groups. ${ }^{26}$

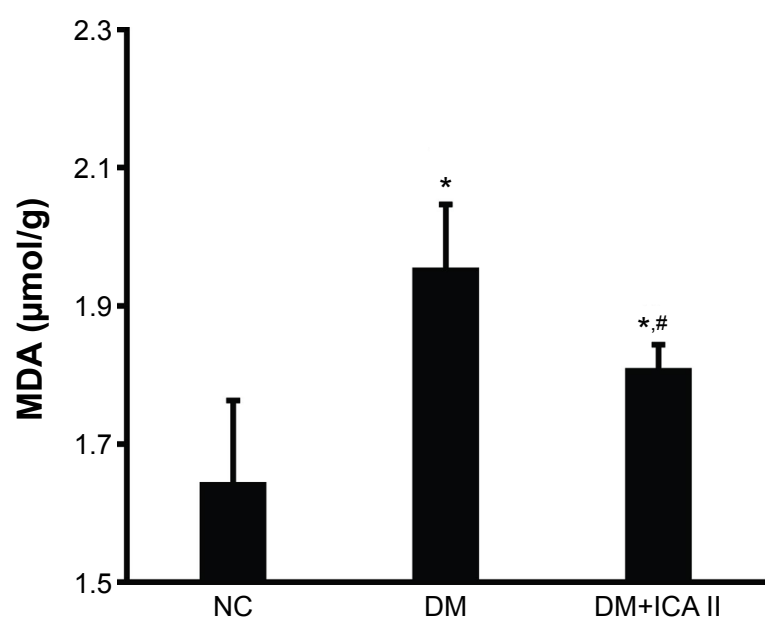

Figure 3 Evaluation of MDA concentrations.

Notes: The MDA concentrations in renal tissues from each group. Quantative data of MDA concentrations in each group are shown in the column bar graph $(n=8$ per group). $* P<0.05$ versus $N C$ group and ${ }^{*} P<0.05$ versus $D M$ group.

Abbreviations: DM, diabetes mellitus; MDA, malondialdehyde; NC, normal control; ICA, icariside.
In the present study, we found that the endothelial content detected by the expression of vWF in glomeruli was significantly reduced in the STZ-induced rats when compared with the NC group. However, the administration of ICA II could significantly increase the endothelial content. Oxidative stress has been found to play an important role in the development and progression of DN, while hyperglycemia is the main cause of the formation of reactive oxygen species, which is involved in the production of oxidative damage. ${ }^{27}$ Excessive oxidative stress in the vascular and cellular milieu results in dysfunction of endothelial cell. Oxidative damage in the STZinduced diabetic rats was ascertained in the present study. Lipid peroxidation was assessed in renal lysate by measuring the MDA level. STZ treatment induced a significant increase in the level of MDA compared with that in the NC group. ICA II treatment could significantly attenuate the MDA levels in the kidney tissues of STZ-induced rats. These results indicated that the antioxidant capacity of ICA II may play an important role in the improvement of epithelial content.

Previous researches have demonstrated that TGF- $\beta$ is one of the most important mediators in the development of 
A

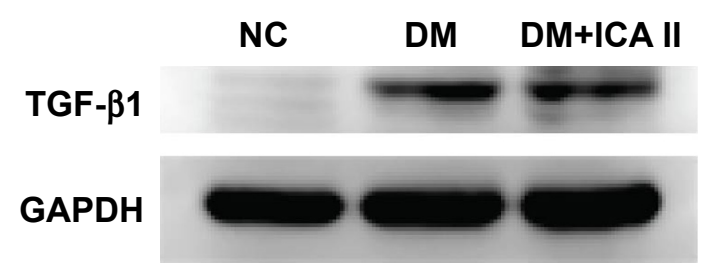

C

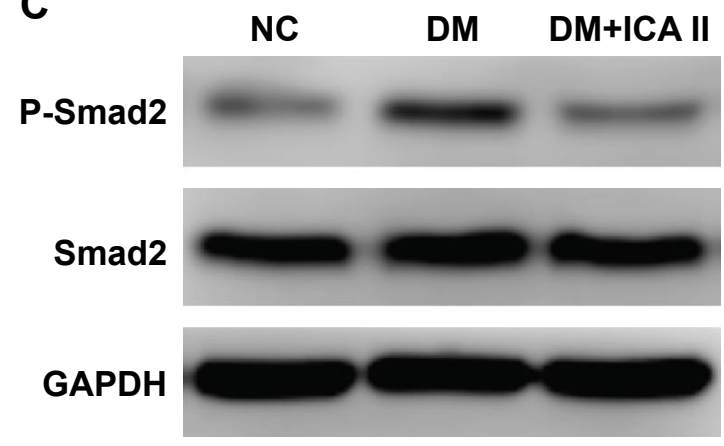

E

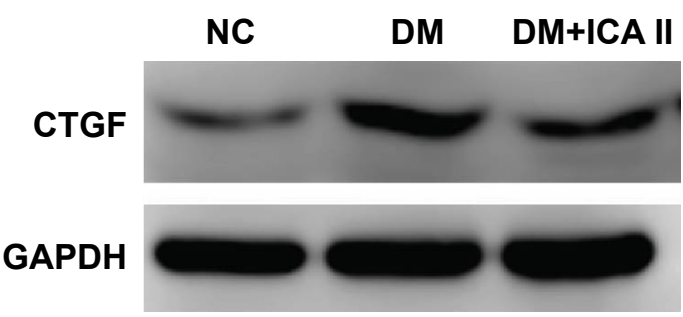

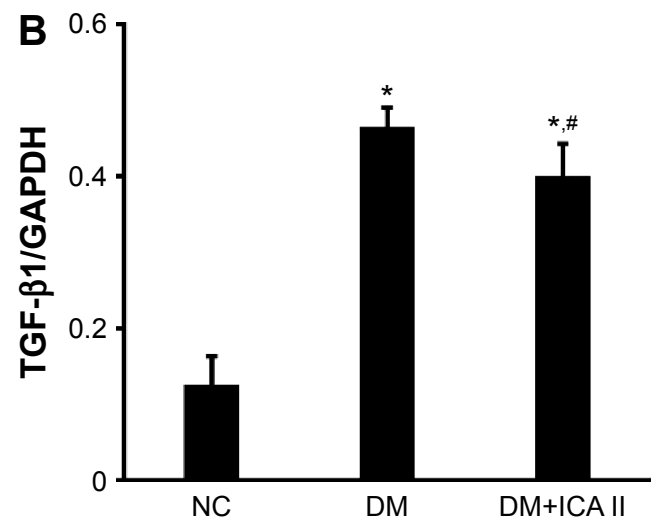
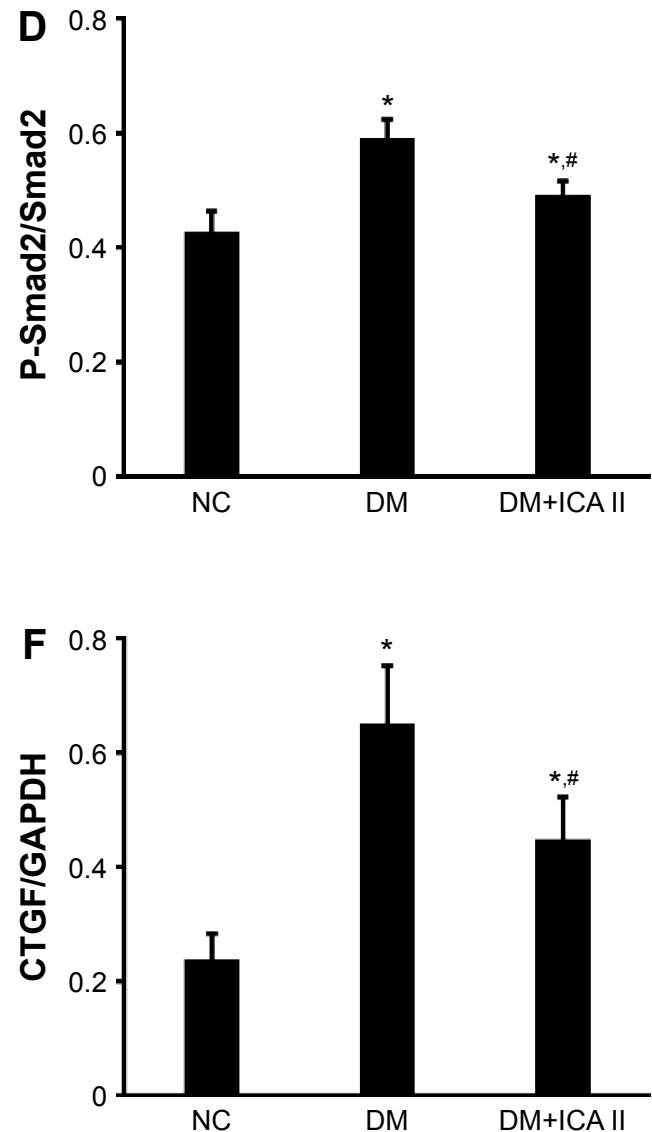

Figure 4 Effects of icariside II on protein levels of TGF- $\beta$ I, P-Smad2, Smad2, and CTGF in kidney.

Notes: (A and B) The protein bands and quantification levels of GAPDH and TGF- $\beta$ I detected by Western blotting; (C and D) the protein bands and quantification levels of GAPDH and P-Smad2, and Smad2 detected by Western blotting; (E and F) the protein bands and quantification levels of GAPDH and CTGF detected by Western blotting. GAPDH was used as the internal standard in each sample. Quantification of protein levels was expressed as mean with standard deviation in each column ( $\mathrm{n}=\mathbf{8}$ per group). $* P<0.05$ versus $\mathrm{NC}$ group and ${ }^{*} P<0.05$ versus $\mathrm{DM}$ group.

Abbreviations: CTGF, connective tissue growth factor; DM, diabetes mellitus; ICA, icariside; NC, normal control; ICA, icariside; Smad, drosophila mothers against decapentaplegic protein; TGF, transforming growth factor.

mesangial dysfunction of $\mathrm{DN},{ }^{10}$ which regulates at least 60 ECM-related downstream genes including Smad and CTGF. Our previous study has showed that TGF- $\beta / \mathrm{Smad} / \mathrm{CTGF}$ pathway involved in the changes of the corpus cavernosum of diabetic rats with erectile dysfunction. ${ }^{28}$ Upregulation of phosphorylation of Smad2 and Smad3 leads to tissue fibrosis in several diseases including diabetes. Furthermore, CTGF intervenes the fibroblast proliferation, migration, adhesion, and ECM deposition. It has also been reported that TGF- $\beta$ inhibited endothelial cell proliferation and migration via the Smad pathway, whereas inhibition of the receptor kinases for TGF- $\beta$ facilitated proliferation of embryonic stem 

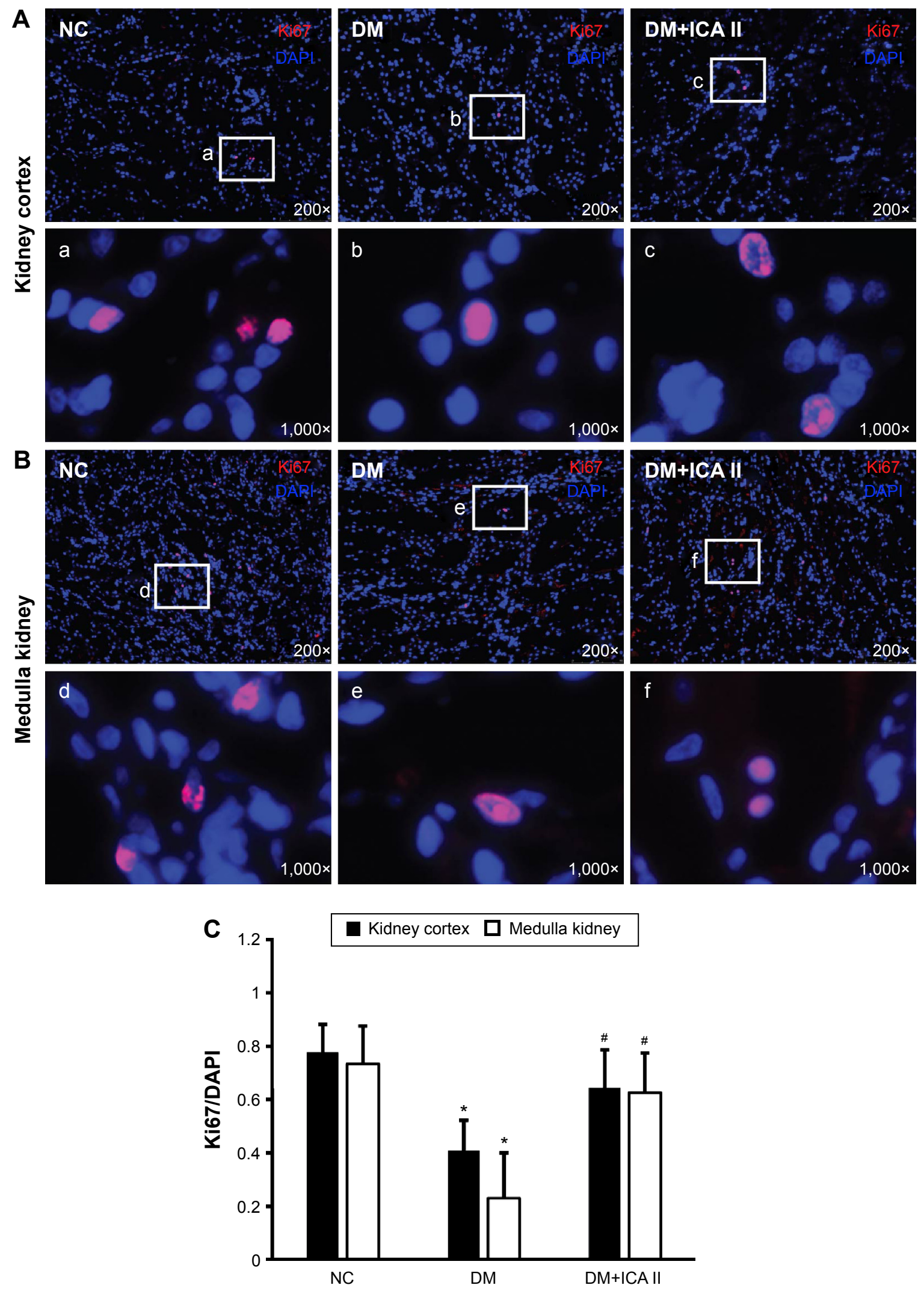

Figure 5 Alteration of Ki67 in kidney cortex and medulla.

Notes: Cell proliferations in kidney cortex and medulla were assessed as expression of Ki67. (A and $\mathbf{B}$ ) The results are shown in the representative histological images

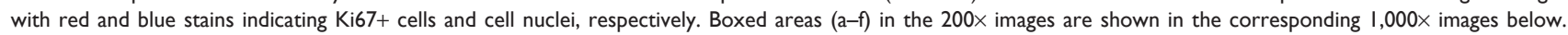
(C) Quantitative data of Ki67+ cells are shown in the bar chart ( $n=8$ per group). ${ }^{*} P<0.05$ versus NC group and ${ }^{\#} P<0.05$ versus $D M$ group.

Abbreviations: DAPI, 4',6-diamidino-2-phenylindole; DM, diabetes mellitus; ICA, icariside; NC, normal control. 

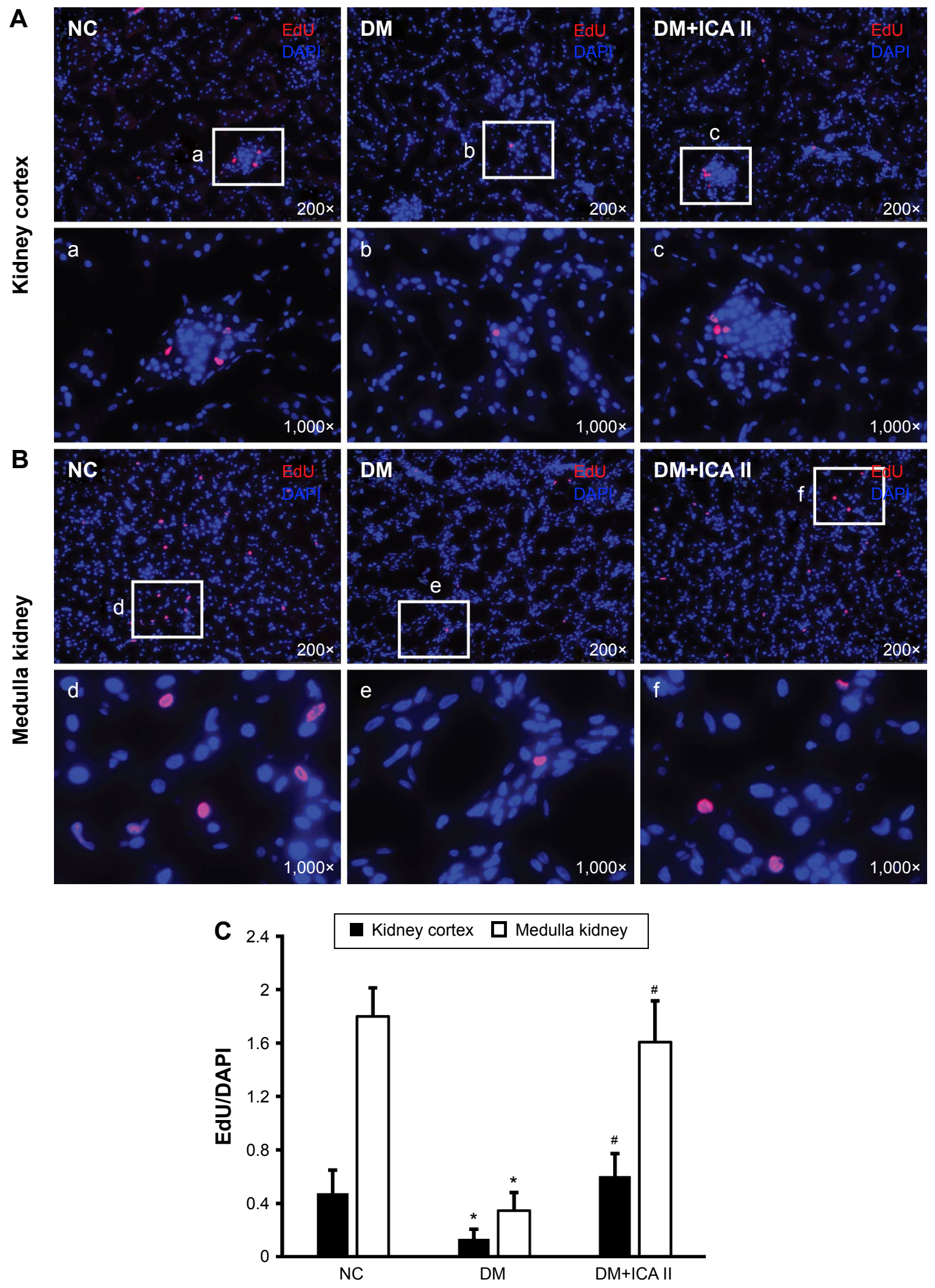

Figure 6 EdU labeling and retaining in renal tissues.

Notes: Rats were intraperitoneally injected with EdU immediately within the first day after birth, and their kidney tissues were examined by fluorescent chemical staining for EdU+ cells at last. (A and $\mathbf{B}$ ) The results are shown in the representative histological images with red and blue stains indicating EdU+ cells and cell nuclei, respectively. Boxed areas $(a-f)$ in the $200 \times$ images are shown in the corresponding I,000x images below. (C) Quantitative data of EdU+ cells are shown in the bar chart ( $\mathrm{n}=8$ per group). $* P<0.05$ versus $\mathrm{NC}$ group and ${ }^{\#} P<0.05$ versus $\mathrm{DM}$ group.

Abbreviations: DAPI, 4',6-diamidino-2-phenylindole; DM, diabetes mellitus; EdU, 5-ethynyl-2-deoxyuridine; ICA, icariside; NC, normal control. 
cell-derived endothelial cells. ${ }^{29,30}$ Hence, TGF- $\beta /$ Smad/CTGF pathway has been recognized as a potential target to prevent or treat the DN. In the present study, TGF- $\beta / \mathrm{Smad} / \mathrm{CTGF}$ signaling pathway was activated in the STZ-induced rats compared with the NC, but it was significantly suppressed when the diabetic rats were treated with ICA II.

EdU, a replacement for BrdU, has recently been used for tracking MSCs. EdU labeling in adipose-derived stem cells (ADSCs) has been confirmed that it was nontoxic to ADSCs and did not interfere with the differentiation, cytokine secretion, or migratory response of ADSCs to stromal cell-derived factor- $1 .{ }^{15} \mathrm{Ki} 67$, a nuclear protein that, is thought to be required for maintaining cell proliferation, is present during all active phases of the cell cycle, and is a cellular marker for proliferation. The present study shows that diabetic rats with ICA II administration had a significantly higher number of Ki67+ cells and EdU+ progenitor cells in both kidney cortex and medulla than those in the DM group, which indicated a possibility of recruitment of progenitor cells. The mechanism of why and what kind of cell was proliferated exactly in the kidney need to be further investigated in future study.

In summary, the present study showed that STZ-induced DM is associated with DN, which shows a decreased epithelial content, activation of TGF- $\beta /$ Smad/CTGF signaling pathway, increased oxidative stress level, and impaired renal function, and ICA II was able to functionally and structurally restore these changes. Furthermore, we also showed that these beneficial effects from ICA II were possibly mediated by its antioxidant capacity and increased recruitment of endogenous stem cells into the kidney tissue. Taken together, these results suggest that ICA II could be a new prospective therapeutic agent for treatment of DN. However, it should be cautioned that the present study is still preliminary and required further validation. Specifically, the identification, properties, and effective mechanism (differentiation or paracrine effects) need to be investigated.

\section{Conclusion}

ICA II treatment significantly ameliorates DN in STZinduced diabetic rats by increasing endothelial cell contents, downregulating TGF- $\beta / \mathrm{Smad} / \mathrm{CTGF}$ signaling pathway and oxidative stress level, and promoting cell proliferation both in kidney cortex and medulla. These beneficial effects appear to be mediated by its antioxidant capacity and recruitment of endogenous EdU+ progenitor cells into the kidney tissue. These results demonstrated that ICA II could be a promising new modality to prevent or postpone DN.

\section{Acknowledgment}

This work was supported by the National Natural Science Foundation of China: No 81270693, No 81272531, and No 81470921.

\section{Disclosure}

The authors report no conflicts of interest in this work.

\section{References}

1. Vleming LJ, Baelde JJ, Westendorp RG, Daha MR, van Es LA, Bruijn JA. The glomerular deposition of PAS positive material correlates with renal function in human kidney diseases. Clin Nephrol. 1997; 47(3):158-167.

2. Ziyadeh FN. The extracellular matrix in diabetic nephropathy. Am J Kidney Dis. 1993;22(5):736-744.

3. Tan AL, Forbes JM, Cooper ME. AGE, RAGE, and ROS in diabetic nephropathy. Semin Nephrol. 2007;27(2):130-143.

4. Wendt T, Tanji N, Guo J, et al. Glucose, glycation, and RAGE: implications for amplification of cellular dysfunction in diabetic nephropathy. J Am Soc Nephrol. 2003;14(5):1383-1395.

5. Zhou X, Wang B, Zhu L, Hao S. A novel improved therapy strategy for diabetic nephropathy: targeting AGEs. Organogenesis. 2012;8(1): $18-21$.

6. Lizicarova D, Krahulec B, Hirnerova E, Gaspar L, Celecova Z. Risk factors in diabetic nephropathy progression at present. Bratisl Lek Listy. 2014; 115(8):517-521.

7. Yamamoto T, Nakamura T, Noble NA, Ruoslahti E, Border WA. Expression of transforming growth factor beta is elevated in human and experimental diabetic nephropathy. Proc Natl Acad Sci U S A. 1993;90(5):1814-1818.

8. Mulay SR, Gaikwad AB, Tikoo K. Combination of aspirin with telmisartan suppresses the augmented TGFbeta/smad signaling during the development of streptozotocin-induced type I diabetic nephropathy. Chem Biol Interact. 2010;185(2):137-142.

9. Brigstock DR. Regulation of angiogenesis and endothelial cell function by connective tissue growth factor (CTGF) and cysteine-rich 61 (CYR61). Angiogenesis. 2002;5(3):153-165.

10. Umezono T, Toyoda M, Kato M, et al. Glomerular expression of CTGF, TGF-beta 1 and type IV collagen in diabetic nephropathy. JNephrol.2006; 19(6):751-757.

11. Abdel Aziz MT, Wassef MA, Ahmed HH, et al. The role of bone marrow derived-mesenchymal stem cells in attenuation of kidney function in rats with diabetic nephropathy. Diabetol Metab Syndr. 2014;6(1):34.

12. Wang S, Li Y, Zhao J, Zhang J, Huang Y. Mesenchymal stem cells ameliorate podocyte injury and proteinuria in a type 1 diabetic nephropathy rat model. Biol Blood Marrow Transplant. 2013;19(4):538-546.

13. Lv SS, Liu G, Wang JP, et al. Mesenchymal stem cells transplantation ameliorates glomerular injury in streptozotocin-induced diabetic nephropathy in rats via inhibiting macrophage infiltration. Int Immunopharmacol. 2013;17(2):275-282.

14. Zhou H, Tian HM, Long Y, et al. Mesenchymal stem cells transplantation mildly ameliorates experimental diabetic nephropathy in rats. Chin Med J (Engl). 2009;122(21):2573-2579.

15. Ning H, Albersen M, Lin G, Lue TF, Lin CS. Effects of EdU labeling on mesenchymal stem cells. Cytotherapy. 2013;15(1):57-63.

16. Zhou F, Xin H, Liu T, et al. Effects of icariside II on improving erectile function in rats with streptozotocin-induced diabetes. J Androl. 2012; 33(5):832-844.

17. Xu Y, Guan R, Lei H, et al. Implications for differentiation of endogenous stem cells: therapeutic effect from icariside II on a rat model of postprostatectomy erectile dysfunction. Stem Cells Dev. 2015;24(6): 747-755.

18. Zhai YK, Guo X, Pan YL, et al. A systematic review of the efficacy and pharmacological profile of herba epimedii in osteoporosis therapy. Pharmazie. 2013;68(9):713-722. 
19. Zhu DY, Lou YJ. Inducible effects of icariin, icaritin, and desmethylicaritin on directional differentiation of embryonic stem cells into cardiomyocytes in vitro. Acta Pharmacol Sin. 2005;26(4):477-485.

20. Li SY, Teh BS, Seow WK, Li F, Thong YH. Effects of the plant flavonoid baohuoside-1 on cancer cells in vitro. Cancer Lett. 1990;53(2-3): 175-181.

21. Wu J, Song T, Liu S, Li X, Li G, Xu J. Icariside II inhibits cell proliferation and induces cell cycle arrest through the ROS-p38-p53 signaling pathway in A375 human melanoma cells. Mol Med Rep. 2015;11(1) 410-416.

22. Qian Q, Li SL, Sun E, et al. Metabolite profiles of icariin in rat plasma by ultra-fast liquid chromatography coupled to triple-quadrupole/time-offlight mass spectrometry. J Pharm Biomed Anal. 2012;66:392-398.

23. Qi MY, Kai C, Liu HR, Su YH, Yu SQ. Protective effect of Icariin on the early stage of experimental diabetic nephropathy induced by streptozotocin via modulating transforming growth factor beta1 and type IV collagen expression in rats. J Ethnopharmacol. 2011;138(3):731-736.

24. Cai WJ, Huang JH, Zhang SQ, et al. Icariin and its derivative icariside II extend healthspan via insulin/IGF-1 pathway in C.elegans. PLoSOne. 2011; 6(12):e28835.
25. Tervaert TW, Mooyaart AL, Amann K, et al. Pathologic classification of diabetic nephropathy. J Am Soc Nephrol. 2010;21(4):556-563.

26. Parvizi MR, Parviz M, Tavangar SM, et al. Protective effect of magnesium on renal function in STZ-induced diabetic rats. J Diabetes Metab Disord. 2014;13(1):84.

27. Dave GS, Kalia K. Hyperglycemia induced oxidative stress in type-1 and type-2 diabetic patients with and without nephropathy. Cell Mol Biol. 2007;53(5):68-78.

28. Zhou F, Li GY, Gao ZZ, et al. The TGF-beta1/Smad/CTGF pathway and corpus cavernosum fibrous-muscular alterations in rats with streptozotocin-induced diabetes. J Androl. 2012;33(4):651-659.

29. Castanares C, Redondo-Horcajo M, Magan-Marchal N, ten Dijke P, Lamas S, Rodriguez-Pascual F. Signaling by ALK5 mediates TGFbeta-induced ET-1 expression in endothelial cells: a role for migration and proliferation. J Cell Sci. 2007;120(Pt 7):1256-1266.

30. Watabe T, Nishihara A, Mishima K, et al. TGF-beta receptor kinase inhibitor enhances growth and integrity of embryonic stem cell-derived endothelial cells. J Cell Biol. 2003;163(6):1303-1311.

\section{Publish your work in this journal}

Drug Design, Development and Therapy is an international, peerreviewed open-access journal that spans the spectrum of drug design and development through to clinical applications. Clinical outcomes, patient safety, and programs for the development and effective, safe, and sustained use of medicines are a feature of the journal, which has also been accepted for indexing on PubMed Central. The manuscript management system is completely online and includes a very quick and fair peer-review system, which is all easy to use. Visit http://www.dovepress.com/testimonials.php to read real quotes from published authors.

Submit your manuscript here: http://www.dovepress.com/drug-design-development-and-therapy-journal 